\title{
Effects of Tinnitus on Quality of Life Among Sawmill Workers
}

\author{
Bright Otoghile $^{1, *}$, Confidence Chioma Otoghile ${ }^{2}$, Nasir Olakunle Ariyibi ${ }^{3}$ \\ ${ }^{1}$ Department of Otorhinolaryngology, Federal Medical Centre, Yenagoa, Nigeria \\ ${ }^{2}$ Department of Psychology, Faculty of Social Sciences, Obafemi Awolowo University, Ile-Ife, Nigeria \\ ${ }^{3}$ Department of Community Medicine, Federal Medical Centre, Yenagoa, Nigeria
}

Email address:

otoghilebright@gmail.com (B. Otoghile)

${ }^{*}$ Corresponding author

\section{To cite this article:}

Bright Otoghile, Confidence Chioma Otoghile, Nasir Olakunle Ariyibi. Effects of Tinnitus on Quality of Life Among Sawmill Workers. International Journal of Otorhinolaryngology. Vol. 4, No. 1, 2018, pp. 31-34. doi: 10.11648/j.ijo.20180401.18

Received: July 21, 2018; Accepted: August 6, 2018; Published: September 1, 2018

\begin{abstract}
Tinnitus is a sensation of sound without an external stimulus and it is a common phenomenon among noise exposed workers. Tinnitus can be very challenging for its sufferers and it could possibly affect their quality of life in various ways. This study aimed to find the effects of tinnitus on quality of life among noise exposed workers. This study was a prospective, community based cross sectional study involving 420 sawmill workers in Ile-Ife. Library and administrative staff of Obafemi Awolowo University, Ile-Ife were used as control and the quality of life of all participants was evaluated with World Health Organization Quality of Life bref questionnaire. Self reported tinnitus severity was evaluated using the Tinnitus Handicap Inventory. Correlation was done between quality of life scores and tinnitus severity scores. The sawmill workers were found to be predominantly males with a male to female ratio of 41: 1 . Out of the 420 sawmill subjects, 41 ( $9.8 \%$ ) had tinnitus and only one of control had tinnitus. There was a negative correlation between tinnitus and the physical, psychological and social domains of quality of life. Increase in tinnitus severity was found to be associated with a significant reduction in physical, psychological and social domains of the quality of life.
\end{abstract}

Keywords: Tinnitus, Noise, Quality of Life, Sawmill Workers

\section{Introduction}

Tinnitus is an abnormal perception of sounds without an external stimulus [1]. It varies in pitch and in its loudness and is usually described as ringing sensation, hissing, buzzing, and clicking sounds. Tinnitus differs from auditory hallucinations which usually occur in patients with central nervous system disorders, psychiatric disorders and alcoholism [2]. This is because unlike auditory hallucination there is absence of organization of its content. This condition could be present with or without hearing loss [3]. Tinnitus could be objective or subjective [4]. Some causes of subjective tinnitus include wax impaction, otitis media, presbyacusis, ototoxic drugs, tumours of the cranial nerve VIII. Some common causes of objective tinnitus include arterio-venous malformations, palatal myoclonus, glomus tumours. It is essential to note that cases of psychogenic tinnitus in which no organic cause is found have also been reported in the literature [5]. Studies have shown that prolonged exposure to loud sound could possibly predispose to tinnitus [6, 7]. Tinnitus often times become chronic and distressing. Hearing an abnormal sound could affect sleep, ability to concentrate in daily activities, performance and could potentially affect the well being of an individual [8]. It could possibly be so disturbing as to cause a spectrum of psychological conditions such as depression, mood problems, emotional problems and even suicidal attempts. Hence, this study aims to find the effects of tinnitus on quality of life among sawmill workers as these workers are particularly exposed to occupational noise.

\section{Methods}

\subsection{Study Design}

This is a comparative cross sectional study involving 
sawmill workers in Ile-Ife. The library and Administrative staff of the Obafemi Awolowo University, Ile-Ife of similar sociodemographic status were used as control. Data was obtained from the sawmill workers and analyzed.

\subsection{Study Setting}

The study was done in Ife East and Ife Central Local Government areas of Osun State in the southwestern part of Nigeria.

\subsection{Study Protocol}

The study involved 420 sawmill workers in Ile-Ife. Also, 420 Library and Administrative staff of similar age and sex of the Obafemi Awolowo University were recruited into the study as control. Inclusion criteria included all adult sawmill workers including male and female from ages of 18 to 60 years who gave consent for the study. Exclusion criteria included any history and examination suggestive of other chronic diseases at the time of recruitment such as such as diabetes mellitus, hypertension, sickle cell disease. Participants were assisted to fill biodata questionnaire. History and severity of self reported tinnitus was collated using Tinnitus Handicap Inventory and the World Health Organization Quality of Life (WHO-QoL) Bref questionnaire was also administered to all the participants. Tinnitus Handicap Inventory was designed by Newman In 1996; it assesses the impact of tinnitus on daily functioning. The questionnaire contains 25 questions on a 3 point Likert scale (4 for a 'yes', 2 for 'sometimes' and 0 for 'no' ) [9]. Tinnitus was graded as: Grade 1 or slight (THI 0-16), Grade 2 or Mild (THI 18-36), Grade 3 or Moderate (THI 38-56), Grade 4 or severe (THI 58-76), Grade 5 or Catastrophic (THI 78-100). The WHO-QoL Bref questionnaire adheres to the WHO's seminal definition of health as "a state of complete physical, mental and social wellbeing and not merely the absence of disease or infirmity." It adopts a multi-dimensional profile of health related quality of life, dividing it into four domains: Physical health (7 items), psychological wellbeing (6 items), social relationships (3 items), and environmental factors ( 8 items). Two additional items assess overall quality-of-life and self-rated health which is categorized as very poor, poor, neither good nor poor, good and very good. Each item is scored on a 5-point scale, where increase in the score corresponds to a better quality of life evaluation [10].

Data was analyzed using Statistical Package for Social
Sciences version 20.0 and a 'p' value of less than 0.05 was accepted as statistically significant. Results were presented in descriptive format.

\subsection{Ethical Consideration}

The study was done in accordance with the ethical standards of the institutional and national research committee and with the 1964 Helsinki declaration and its later amendments or comparable ethical standards.

The consent of the participants taking part in the study was obtained.

\section{Results}

There were 420 sawmill workers recruited into the study out of which 410 were males and 10 were females with control of same age and sex. The mean age was $34.53 \pm 8.59$ years for the sawmill subjects and $34.61 \pm 8.79$ years for the control group $(\mathrm{t}=1.01, \mathrm{P}=0.285)$. Out of the 420 sawmill subjects, $41(9.8 \%)$ had tinnitus and only one of control had tinnitus. The mean quality of life scores were $65.10 \pm 10.84$ and $73.97 \pm 6.13$ for the sawmill workers and control group respectively. There was a significant difference between the quality of life of the sawmill workers and the control group $(\mathrm{P}<0.05)$. The mean tinnitus score for the sawmill workers was $19.80 \pm$ 2.89. Severity of tinnitus is shown in Table 1.

According to the WHOQoL-bref questionnaire, overall quality of life was classified as very poor, poor, neither poor nor good, good and very good. Greater percentage of the control subjects had good and very good quality of life when compared with the sawmill subjects as shown in Figure 1. There was a significant reduction in the physical, psychological and social domains of the quality of life scores with presence of tinnitus and no significant change in the overall and environmental domains of quality of life with increase in tinnitus severity. This is shown in Table 2.

Table 1. Severity of tinnitus among sawmill workers.

\begin{tabular}{lll}
\hline Tinnitus Grading & Frequency & Percentage \\
\hline Slight & 27 & 65.9 \\
Mild & 9 & 22.0 \\
Moderate & 3 & 7.2 \\
Severe & 0 & 0 \\
Catastrophic & 2 & 4.9 \\
\hline
\end{tabular}

Table 2. Correlation between Tinnitus severity and Quality of life Scores among sawmill workers.

\begin{tabular}{lll}
\hline Variable & N & Correlation coefficient ( r) \\
\hline Overall Quality of Life and tinnitus severity & 41 & -0.257 \\
Physical Quality of Life and tinnitus severity & 41 & -0.492 \\
Psychological Quality of Life and tinnitus severity & 41 & -0.319 \\
Social Quality of Life and tinnitus severity & 41 & -0.380 \\
Environmental Quality of Life and tinnitus severity & 41 & 0.005 \\
\hline
\end{tabular}

Key: $r$ means coefficient of correlation 


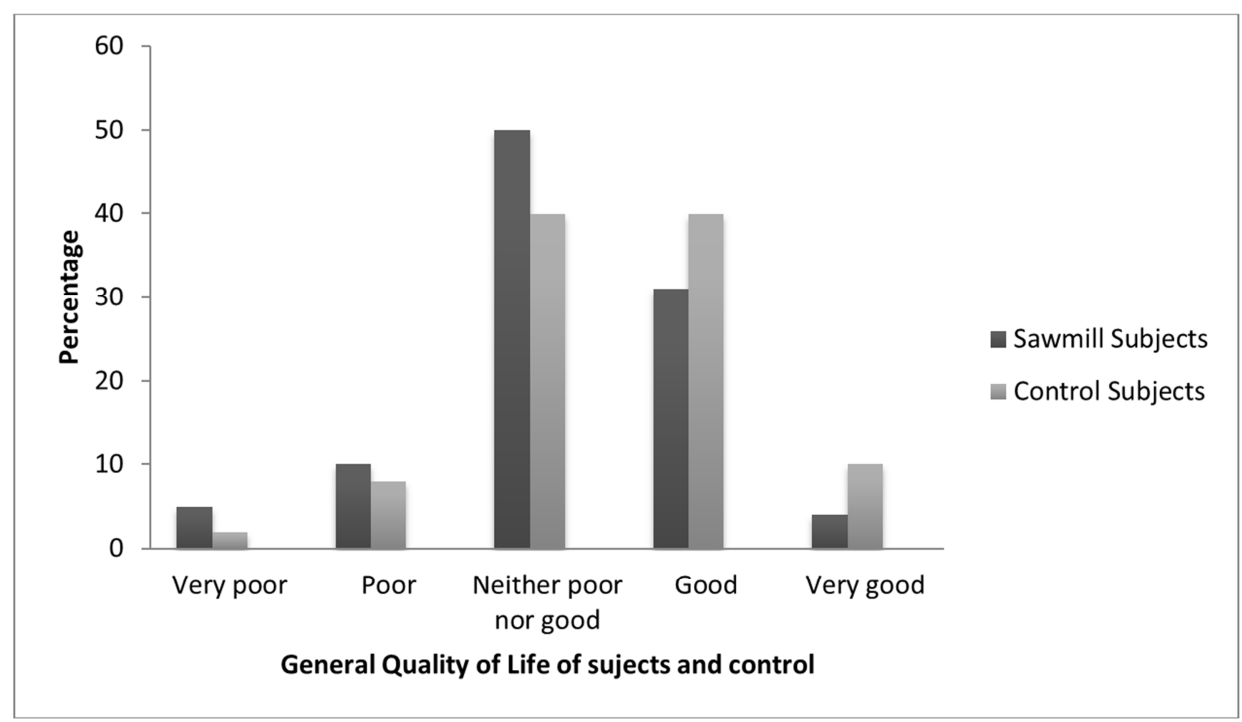

Figure 1. Showing the overall quality of life of subjects and control.

\section{Discussion}

Tinnitus has been found to be a common complaint in the adult population [11]. In this study, $9.8 \%$ of the sawmill workers had tinnitus. Adegbenro et al [12] found a lower prevalence of $6.1 \%$ in the general population in Ile-Ife which is a similar location where this study was done. Hence, tinnitus may be commoner among people exposed to loud noise when compared to the general population. Tinnitus can result from different causes, although it most commonly results from otological causes. According to Axelsson and Prasher [13], noise exposure is the most common cause of tinnitus. Tinnitus can cause a wide range of psychological and somatic distress which have the potential to consequently affect quality of life in various ways. The effects of tinnitus could be psychological, social and physical. This makes the WHO bref quality of questionnaire suitable in this study. The quality of life response of the participants was evaluated as psychological, social, physical and environmental according to the definition of health. Tinnitus being a subjective phenomenon is very difficult to measure and quantify objectively, hence its assessment in this study was done with a questionnaire to obtain data based on the response and description from the participants. The average tinnitus score among the sawmill workers was 19.8. This shows majority of the workers had mild tinnitus. This level of tinnitus can affect sleep which is an important part of daily activity.

Although, there is paucity of comparative data on the level or grade of tinnitus among sawmill workers, tinnitus is however known to affect physical activities such as sleep, alertness, performance $[14,15]$. This study found that increase in tinnitus severity is associated with a reduction in the physical domain of the quality of life. Folmer [16] also reported that tinnitus could cause insomnia. Adequate sleep has been identified as very important to maintain day time alertness which is necessary for good performance [17]. This is particularly important in the sawmill since the work is physically tasking. Poor performance has negative effects on quality of life. Reduction in the physical aspect of quality of life which could possibly be in form of poor performance, sleep, alertness and this is very significant in the sawmill for good productivity. Increase in productivity in the saw mills could contribute to the national economy. Good performance and alertness in the sawmill could also help to reduce injuries among the workers as some of the possible injuries could be life threatening.

Tinnitus severity adversely affects the psychological and social domains of the quality of life as observed in this study. Perception of abnormal sounds as in cases of tinnitus could affect some psychological variables such depression, anxiety and general well being. Adoga and Obindo [18] found that tinnitus could result in anxiety and depression. Also, Folmer et al [19] found out that tinnitus severity positively correlated with measures of anxiety and depression. Sawmill workers need to be very stable psychologically to avoid unnecessary mistakes at work since they use heavy duty machines that require being careful to prevent physical injuries. Hence, the psychological effect of tinnitus should not be ignored. The effects of tinnitus also extend to social life. This could influence interactions within the work place which is important in maintaining industrial harmony.

However, there was no significant relationship between tinnitus severity and the environmental domain of quality of life. There was also no significant relationship between tinnitus severity and overall quality of life in this study. Tiexeral et al [20] and Roggerone et al [21] also observed no significant change in the quality of life among subjects with tinnitus. However, higher levels of quality of life (Good and very good) were reduced among sawmill workers when compared to the control (Figure 1). Tinnitus has therefore been shown to variously affect quality of life.

\section{Conclusion}

The prevalence of tinnitus from this study was found to be $9.80 \%$ and increase in tinnitus severity was found to be 
associated with a significant reduction in physical, psychological and social domains of the quality of life.

We recommend hearing conservation programmes for sawmill workers which will help to reduce the effects of exposure to loud noise. Those sawmill workers already having tinnitus should endeavour to go for treatment.

\section{Conflict of Interest}

All authors declare that there are no conflicts of interest

\section{References}

[1] Levine RA, Oron Y. Tinnitus. In: Handbook of Clinical Neurology. 2015; 409-431.

[2] Ceranic, B. and L. M. Luxon, Tinnitus and Other Dysacusis. In: Scott-Brown's Otorhinolaryngology, Head and Neck Surgery. M. Gleeson, et al (eds). $7^{\text {th }}$. Edward Arnold Ltd: London. 2008; 3594-3628.

[3] M cKee GJ, Stephens SD. An investigation of normally hearing subject with tinnitus. Audiology 1992; 31:313-317.

[4] Baguley D, McFerran D, Hall D. "Tinnitus". The Lancet. 2013; 1600-1607.

[5] Tomoaki KAI. Two cases of Psychogenic Hearing loss in Adults. Practica Oto-Rhino- Laryngologica. 2002; 95: 679684.

[6] Otoghile B, Onakoya PA, Otoghile C. Auditory effects of noise and its prevalence among sawmill workers. Int $J$ Med Med Sc. 2018; 10: 27- 30.

[7] Phoon WH, Lee HS, Chia SE. Tinnitus in noise-exposed workers. Occup Med. 1993; 43: 35-38.

[8] Nondahl DM, Cruickshanks KJ, Dalton DS, Klein BEK, Klein $\mathrm{R}$, Schubert CR, Tweed TS et al. The Impact of Tinnitus on Quality of Life in Older Adults. J Am Acad Audiol. 2007; 18: 257- 266.

[9] Newman CW, Jacobson GP, Spitzer JB. Development of the Tinnitus Handicap Inventory. Arch Otolaryngol Head Neck Surg. 1996; 122:143-148.
[10] Skevington SM, Lotfy M, O'Connell KA. The World Health Organization's WHOQOL-BREF quality of life assessment: psychometric properties and results of the international field trial. A report from the WHOQOL group. Qual Life Res. 2004; 13: 299-310.

[11] Axelsson A, Ringdal A. Tinnitus: a study of its prevalence and characteristics. Br J Audiol 1989; 23:53-62.

[12] Adegbenro CA, Amusa YB, Ijadunola IKT, Adeyemo A. Prevalence of Tinnitus among Nigerians. J Com Med Health Educ. 2013; 3: 200.

[13] Axelsson A, Prasher D. Tinnitus induced by occupational and leisure noise. Noise Health. 2000; 2: 47-54.

[14] Alster J, Shemesh Z, Ornan M, Attias J. Sleep disturbance associated with chronic tinnitus. Bio Psychiatry. 1993; 34: 8490 .

[15] Langguth B. A review of tinnitus symptoms beyond 'ringing in the ears': a call to action. Curr Med Res Opin. 2011; 27: 1635-1643.

[16] Folmer RL, Griest SE. Tinnitus and insomnia. Am $J$ Otolaryngol. 2000; 21: 287-293.

[17] Muzet A. Environmental noise, sleep and health. Sleep Med Rev 2007; 11: 135-142.

[18] Adoga AA, Adoga AS, Obindo JT. Tinnitus and the prevalence of co-morbid psychological stress. Niger J Med. 2008; 17: 95-97.

[19] Folmer RL, Griest SE, Meikle MB, Martin WH. Tinnitus severity, loudness and depression. Otolaryngol Head Neck Surg. 1999; 121: 48-51.

[20] Teixeira AR, Nunes MGP, Freitas CLR, Gonçalves K, Teixeira SB. Analysis of Quality of Life of Seniors with Tinnitus' Symptoms. Int Arch Otorhinolaryngol. 2010; 14:54-59.

[21] Roggerone M. Determinant of Tinnitus' impact in Quality of life in an outpatient Clinic Protocol. MSC Thesis. Groningen. University of Groningen; 2010. 